\title{
Assembly of a Pulmonary Artery Pressure Sensor System
}

\author{
J. Müntjes, S. Meine, E. Flach, M. Görtz, R. Hartmann, \\ T. Schmitz-Rode, H. K. Trieu, W. Mokwa
}

\begin{abstract}
This paper presents an implantable system for telemonitoring the intravascular pressure in the pulmonary artery. By implanting a catheter-bound pressure and temperature sensor into the pulmonary artery, it is possible to monitor the actual value and the time variations of the intravascular pressure with a frequency of $128 \mathrm{~Hz}$. Thus hospitalization of patients suffering from heart insufficiency can be avoided by early changes in therapy.

Preliminary in vivo experiments have been conducted to verify the fixation mechanism and the positioning of the sensor at the right place in the pulmonary artery. It was shown that the proposed fixation mechanism and the packaging of the sensor promise to be stable.
\end{abstract}

Keywords: heart insufficiency, pressure sensor, microsystems technology, assembly.

\section{Introduction}

A considerable number of people in Europe suffer from congestive heart failure. In Germany, the number is around 1.8 million, rising by 2-300000 each year [1]. This poses a substantial economic problem. In Fig. 1, the occurrence of heart insufficiency is compared to that of other reasons for hospitalization [2]. As it is one of the most frequent non-surgical diseases with recurring hospitalization and high mortality, a strategy to lower the costs is required.

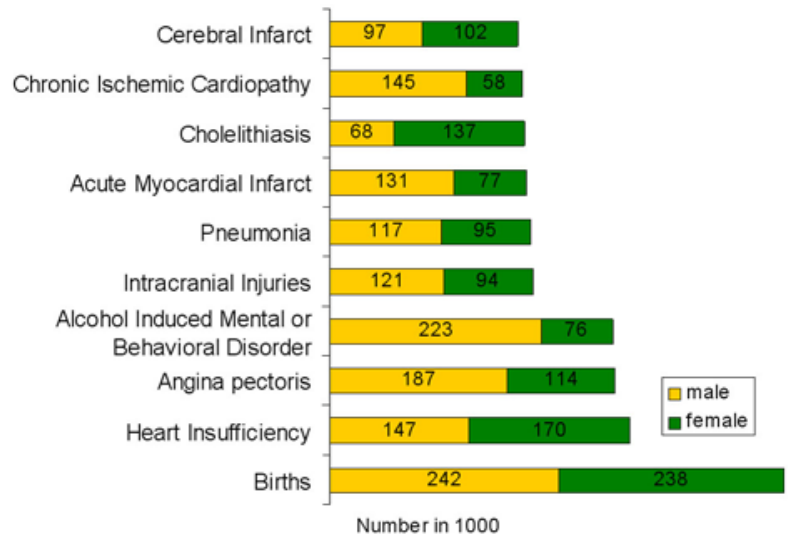

Fig. 1: Reasons for Hospitalization in Germany 2006 [2]

Patients can be monitored by measuring the pulmonary artery pressure and deriving the cardiac output by means of a modified pulse contour analysis. This provides information about the physical health of the patient, and serves as an early warning system for the physician. Fig. 2 shows the pressure change over time before hospitalization where the degradation is measured and gives information about the health state of the patient. Risk of infection, however, would be given if parts of the monitoring system were situ- ated outside the body. Therefore, a fully implantable system is presented which includes a catheter-bound pressure and temperature sensor inside the pulmonary artery. The system enables the physician to monitor the actual value and the time variations of the intravascular pressure with a frequency of $128 \mathrm{~Hz}$. Thus hospitalization of patients suffering from heart insufficiency can be avoided by early changes in therapy.

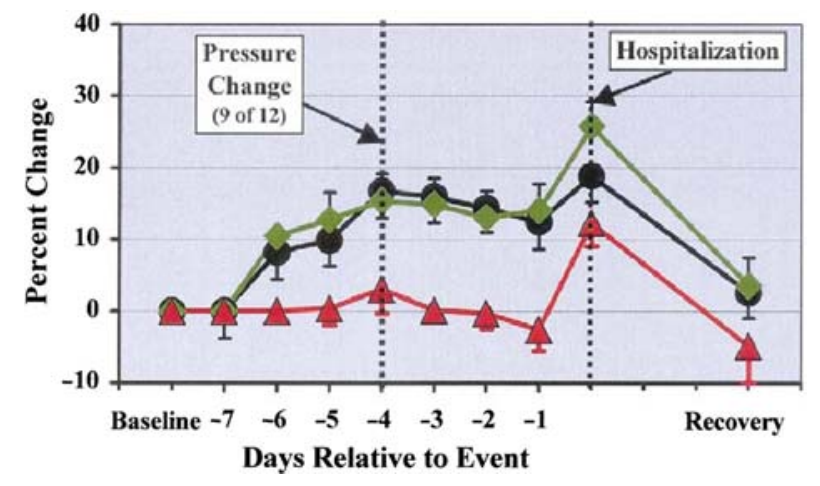

Fig. 2: Pressure Changes Occurred in 9 of 12 Events 4 2 Days before Hospitalization (major) [3]

\section{Design and fabrication}

The system is the result of cooperation between a medical company and several university institutes. Research at the Institute of Materials in Electrical Engineering covers the assembly process of the pulmonary artery pressure sensor. This paper therefore focuses on the assembly aspects of the sensor system.

\section{$2.1 \quad$ Design}

The system, as shown in Fig. 3, consists of intraand extracorporeal parts. The implanted parts are 
(1) a pressure and temperature sensor enclosed in a metal tube forming the encapsulation, and (2) implanted electronics protected by a biocompatible housing. The sensor is located inside a catheter, which holds the sensor cable connecting the sensor element and the implant. In addition to the interface to the pressure sensor, the implanted electronics contains a telemetric unit, a battery, a processor and an internal memory. Outside the body, a home monitoring station (3) receives the data from the subcutaneous implanted electronics, which is able to communicate wirelessly. The data is then transferred by mobile communication via a service center to the attending physician.

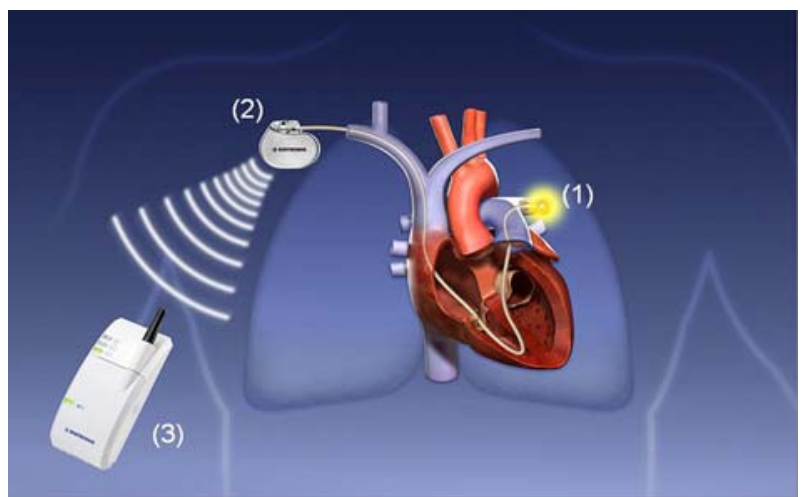

Fig. 3: COMPASS System (with (1) Sensor Element inside the Pulmonary Artery, (2) Implanted Electronics in a Biocompatible Housing and (3) Home Monitoring Station)

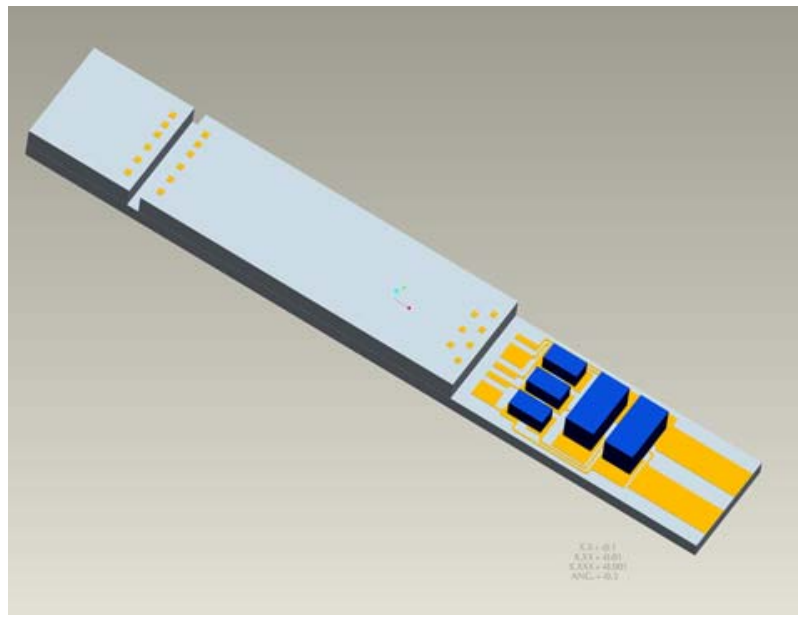

Fig. 4: Sensor Element (Carrier with Pressure Sensor and Signal Processing Chip plus Additional Electronic Devices)

The sensor element, as shown in Fig. 4, contains electronic devices to support the transmission between the sensor element and the implanted electronics and two silicon chips. One is a monolithically integrated pressure and temperature sensor (Fig. 5), similar to the sensor presented in [4]. The circles that are visible on the chip layout represent the capacitive pressure sensors. The other chip is an additional interface circuit amplifying the signal obtained from the sensors.
This allows signal transmission over a long distance between the sensor and the telemetric unit. Both chips are mounted on a carrier and are electrically connected via wire bonds.

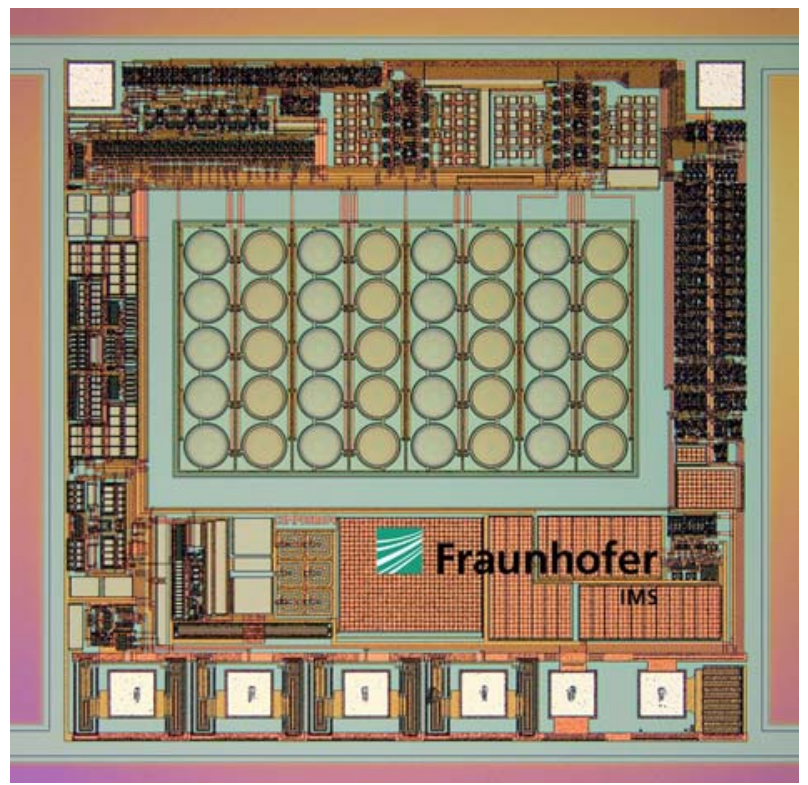

Fig. 5: Monolithically Integrated Capacitive Pressure and Temperature Sensor

\subsection{Fabrication}

Stress-free assembly of the components is crucial to the system: The monolithically fabricated capacitive pressure sensor is very sensitive towards pre-stress. Therefore the pressure sensor and the interface circuit are mounted on a carrier to which the cable running through the catheter can be attached. This carrier is designed to minimize the influence of temperature during operation inside the body. Silicon is chosen as the carrier material because of its identical thermal expansion coefficient. Fig. 6 shows the design of the silicon carrier. Because of the limited space inside the metal sensor capsule, it is crucial to design the circuit lines with the smallest possible pitch. To assist the signal processing chip, three capacitors and two diodes in surface-mount technology are additionally soldered to the carrier. Again the limited space inside the metal encapsulation has to be taken into account, and it is for this reason that small-scale SMT devices have to be used.

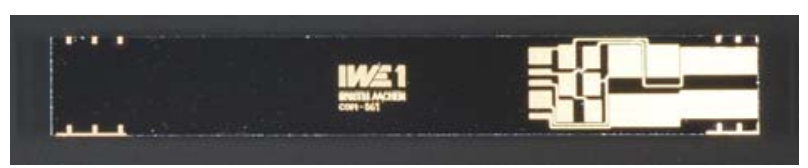

Fig. 6: Silicon Carrier

A single step process using flexible adhesive was developed to attach both silicon chips to the carrier. 
This flexibility is needed to reduce stress on the capacitive pressure sensor [5]. The low-viscosity adhesive fills the gaps between the two chips without any bubbles, see Fig. 7. This is important in order to ensure drift-reduced operation of the sensor assembly.

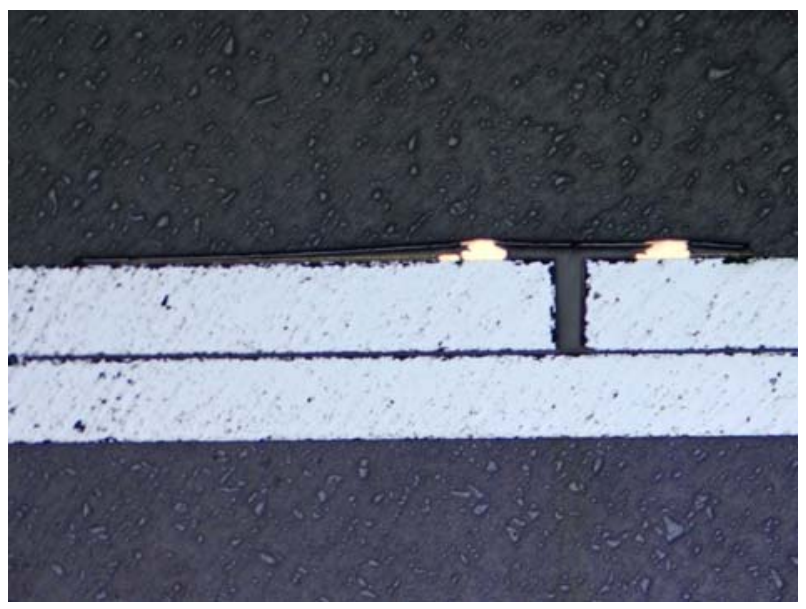

Fig. 7: Sensor Assembly after the Single Step Adhesion Process, Cross Section

Fig. 8 shows the three fabrication stages of the assembly: (1) the carrier with additional SMT devices after the single step adhesion process, (2) the signal processing chip is bonded to the carrier by wire bonding and (3) the complete structure provided with a glob-top adhesive to protect the thin wire bonds. Dummy chips are used instead of a pressure sensor and a signal processing chip. To protect the assembly against the highly aggressive environment which human blood poses to electronics, a metal tube forms the encapsulation. This tube is opened above the pressure sensor to allow the blood pressure to be measured. The tube itself is filled with an incompressible medium to ensure good pressure propagation towards the sensor.

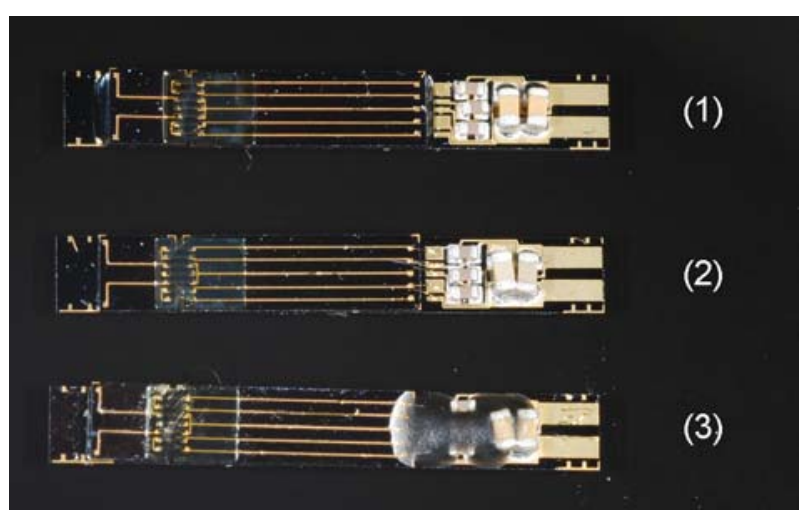

Fig. 8: Fabrication Stages of the Sensor Assembly (Dummy Chips; (1) Chip Carrier with SMT Devices after the Single Step Adhesion Process, (2) after Wire Bonding, (3) after Glob-Top)
Fig. 9 shows the metal pins to which the cable can be connected. The pins are soldered to the two long gold pads on the right of the sensor carrier. A ceramic connector then forms the closure of the metal tube. This decouples the pressure sensor from the stress induced by movements of the catheter inside the blood vessel, and at the same time relieves the strain. The complete system is integrated in a catheter $80 \mathrm{~cm}$ in length. Its distal end is fixed in the pulmonary artery; the proximal end of the catheter is attached to the telemetric unit.

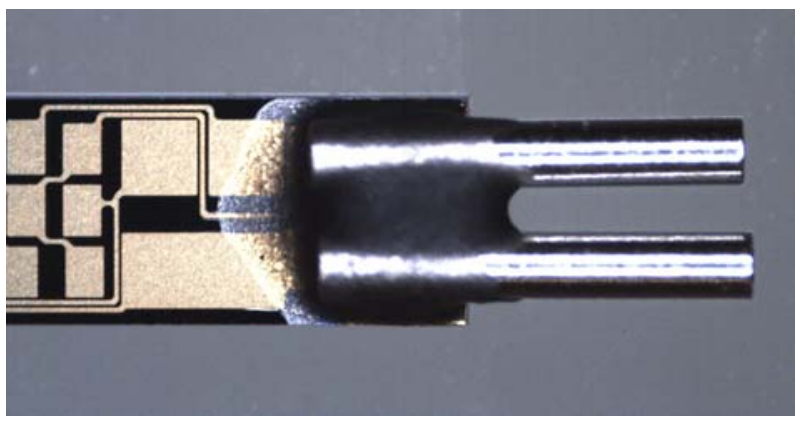

Fig. 9: Metal Connectors to Sensor Cable

\section{Experiments and discussion}

The silicon carrier, which had been thinned to fulfil the geometric requirements, was subjected to several shear tests. With the ceramic connector stabilizing the sensor element, a breaking point could be ascertained that was high enough to assure stability during the handling and operation process. The first in vivo experiments have already been conducted to verify the fixation mechanism and the positioning of the sensor at the right place in the pulmonary artery. It was shown that the proposed fixation mechanism and the packaging of the sensor promise to be stable. Long-term in vivo experiments will be conducted in the near future.

\section{Conclusion}

A new implantable pressure and temperature sensor system for monitoring pulmonary artery pressure and cardiac output has been presented. The assembly process promises to be stable and is expected to impose little stress on the capacitive pressure sensor measuring the pulmonary artery pressure. By avoiding stress on the sensor, the measurements will give stable results and the drift will be reduced to a minimum.

\section{Acknowledgement}

The research described in the paper was supervised by Prof. W. Mokwa, RWTH Aachen University and was supported by the German Federal Ministry of Education and Research (BMBF), grant COMPASS. 


\section{References}

[1] Fischer, M., et al.: Epidemiologie der linksventrikulären systolischen Dysfunktion in der Allgemeinbevölkerung Deutschlands, Zeitschrift Fuer Kardiologie, 92, 4, 294-302.

[2] Statistisches Bundesamt: Diagnosedaten der Patienten und Patientinnen in Krankenhäusern (einschl. Sterbe- und Stundenfälle).

[3] Adamson, P. B, et al.: Ongoing right ventricular hemodynamics in heart failure: clinical value of measurements derived from an implantable monitoring system., J. AM. Coll Cardiol, 41, 4, $565-571$.

[4] Fassbender, H., et al.: Fully implantable blood pressure sensor for hypertonic patients, The Seventh IEEE Conference on SENSORS, 1226-1229.

[5] Wilde, J., Deier, E.: Thermomechanische Einflüsse der Chipklebung auf die Genauigkeit mikromechanischer Drucksensoren, Teil 1: Simulation, Technisches Messen, 70, 5, 251-257.

\section{About the author}

Jutta MÜNTJES is currently working at the Institute of Materials in Electrical Engineering at RWTH Aachen University. Her work focuses on assembly technologies in the field of pressure sensor systems in medical technology. She studied electrical engineering at the University of Erlangen-Nürnberg and at KTH Stockholm, focusing on microelectronics and microsystems technology.
Jutta Müntjes

E-mail: jutta.muentjes@rwth-aachen.de

RWTH Aachen University

Institute of Materials in Electrical Engineering 1

Aachen, Germany

Sonja Meine

Biotronik SE \& Co. KG, Berlin, Germany

Erhard Flach

Biotronik SE \& Co. KG, Berlin, Germany

Michael Görtz

Fraunhofer Institute for Microelectronic Circuits and Systems

Duisburg, Germany

Renate Hartmann

Helmholtz Institute

Department of Applied Medical Engineering

Aachen, Germany

Thomas Schmitz-Rode

Helmholtz Institute

Department of Applied Medical Engineering

Aachen, Germany

Hoc Khiem Trieu

Fraunhofer Institute for Microelectronic Circuits and Systems

Duisburg, Germany

Wilfried Mokwa

RWTH Aachen University

Institute of Materials in Electrical Engineering 1

Aachen, Germany 